\title{
CUIDADO E IDENTIDAD DE GÉNERO \\ De las madres que trabajan a los hombres que cuidan
}

\author{
CARE AND GENDER IDENTITY \\ From working mothers to caring men
}

Constanza Tobío ctobio@polsoc.uc3m.es

Universidad Carlos III de Madrid. España

\begin{abstract}
ResUmen
El cuidado de las personas emerge como un nuevo ámbito de la realidad y campo de conocimiento, hasta ahora invisible. Desde la teoría feminista se identifican las prácticas sociales complejas en que consiste, así como la ética de la responsabilidad en que se sustenta, construida, según Gilligan, a lo largo del proceso de socialización femenino. Al incorporarse los hombres al cuidado se replantea la especificidad femenina de tal actividad y se abren nuevos interrogantes acerca de la identidad de género. Por otra parte, el carácter dicotómico del género se pone en cuestión desde la perspectiva de autores como Fausto-Esterling o Butler, problematizando no solo lo femenino sino también lo masculino. Si en los años cuarenta del pasado siglo Simone de Beauvoir se adelanta al constructivismo sociológico preguntándose cómo se hace una mujer, a comienzos del siglo XXI cobra sentido preguntarse qué es un hombre.
\end{abstract}

\section{Palabras Clave}

Cuidado; Género; Masculinidad.

\begin{abstract}
Care has appeared as a new dimension of social reality and research field, until not so long ago invisible. Feminist theory has identified the complex social practices it is made of as well as the ethics of responsibility which underpin it, all of it, according to Gilligan, constructed through the long process of women's socialization. When men become involved in care, its female specificity is challenged and new questions on gender identity arise. On the other hand the dichotomous character of gender is discussed by authors like Fausto Esterling or Butler; not just women but also men are problematised. If in the forties of the past century Simone de Beauvoir goes ahead sociological constructivism when she asks how is a woman made, at the beginning of the XXI it makes sense to ask what is a man.
\end{abstract}

\section{KEYWORDS}

Care; Gender; Masculinity. 


\section{INTRODUCCIÓN}

Que las mujeres, la mayoría de las mujeres, incluso las madres, trabajen constituye una nueva normalidad social. No hace tanto que la actividad laboral femenina se asociaba a una pauta específica caracterizada por un máximo en las edades más jóvenes seguido de un fuerte descenso coincidiendo con el matrimonio y los hijos. En nuestro país el proceso ha sido especialmente rápido. Desde los años setenta la tasa de actividad se ha duplicado y hoy cuatro de cada cinco mujeres de 25 a 35 años están en el mercado de trabajo (Instituto Nacional de Estadística 2009). El cambio es especialmente destacable entre las españolas de 35 años, la mayor parte de las cuales son madres. Si en 1977 menos del 30\% eran activas, hoy ese porcentaje se eleva al $80 \%$, solo algo menos que la tasa masculina (gráfico 1). El grupo central de edad, de 25-49 años, ha experimentado un incremento similar entre las mujeres, mientras que en el caso de los hombres ha disminuido algo, tal como ocurre en el conjunto de edades (gráfico 2).

Pero afirmar que las mujeres trabajan no deja de ser discutible. Decir que alguien trabaja significa en el lenguaje corriente que lo hace en ciertas condiciones, a cambio de una remuneración y en el marco de una relación explícita de intercambio mercantil, entre otras cosas. De forma metonímica se opone a otro trabajo que se califica como "no trabajo" cuya identificación procede de quién lo hace -una mujer- y en qué marco -el familiar. Las amas de casa, así, resulta que no trabajan o lo hacen en ciertas actividades, "sus labores", que no son propiamente trabajo, trabajo-trabajo, trabajo de verdad.

Hoy que la mayoría de las mujeres "trabajan" en la acepción que el sentido común generalizado da al término, siguen haciéndolo en el "otro trabajo", ese que se entiende como "no trabajo", en un segundo turno (Hochschild 1989) que las encuestas sobre uso del tiempo cuantifican. España e Italia son los países europeos con mayores diferencias en la dedicación de mujeres y hombres a los trabajos domésticos (Eurostat 2006), incluso si las mujeres tienen un empleo, doblando en este caso la dedicación femenina a la masculina, lo cual se acentúa entre los casados, 5 horas y 53 minutos ellas, frente a 2 horas y 22 minutos ellos (Instituto Nacional de Estadística 2003). A diferencia de lo que ocurre con la actividad laboral femenina, la actividad doméstica de los hombres en los últimos años se ha incrementado muy levemente (gráfico 3), al tiempo que las horas de dedicación de las mujeres han disminuido algo, pero muy poco. Las mujeres españolas, según datos del año 2009, dedican la mayor parte de su tiempo activo al hogar y la familia (más de cuatro horas en un día promedio), mientras que los hombres lo hacen al empleo (algo más de tres horas en promedio). Son significativas también las diferencias en dedicación a la vida social, la diversión, los deportes y las aficiones que ocupan dos horas y media diarias a hombres frente a menos de dos horas entre las mujeres (gráfico 4). Ello supone que si se agrega el trabajo laboral y doméstico de hombres y mujeres, estas dedican más tiempo al trabajo 
Gráfico 1.

Tasas de actividad según la edad, por sexo. España, 1977-2008

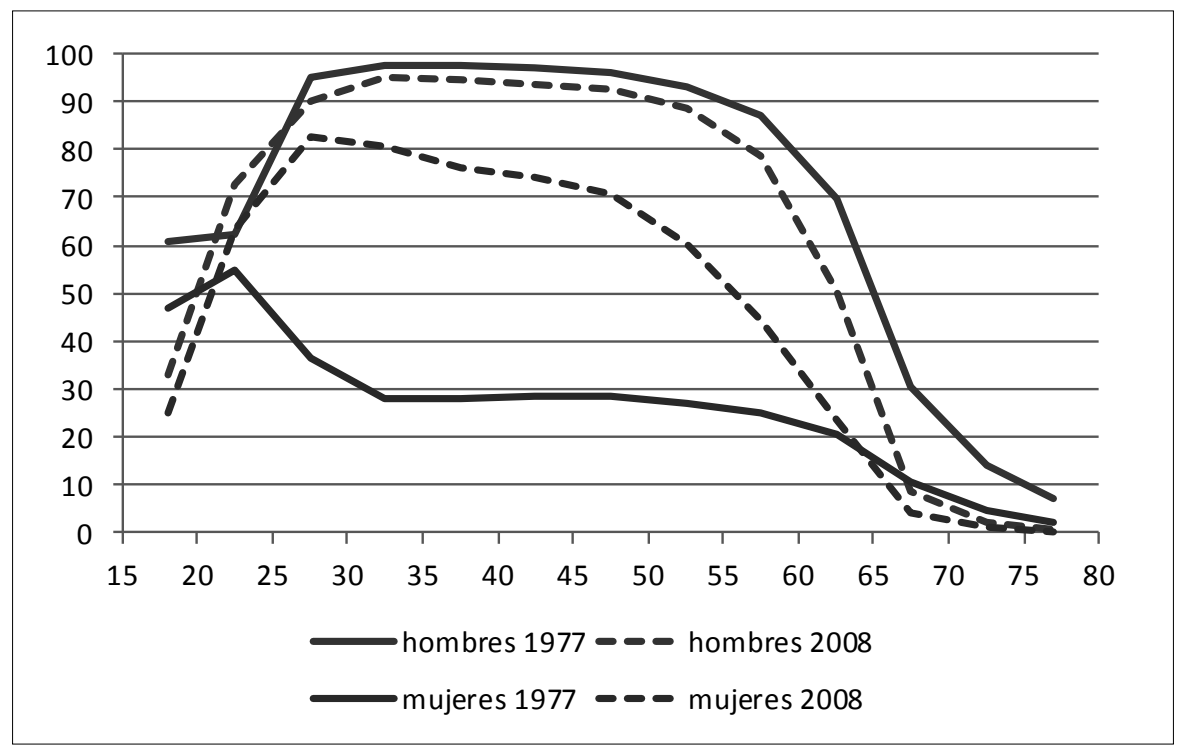

Fuente: Datos de la OIT.

Gráfico 2.

Evolución de la tasa de actividad de hombres y mujeres entre 25 y 49 años. España

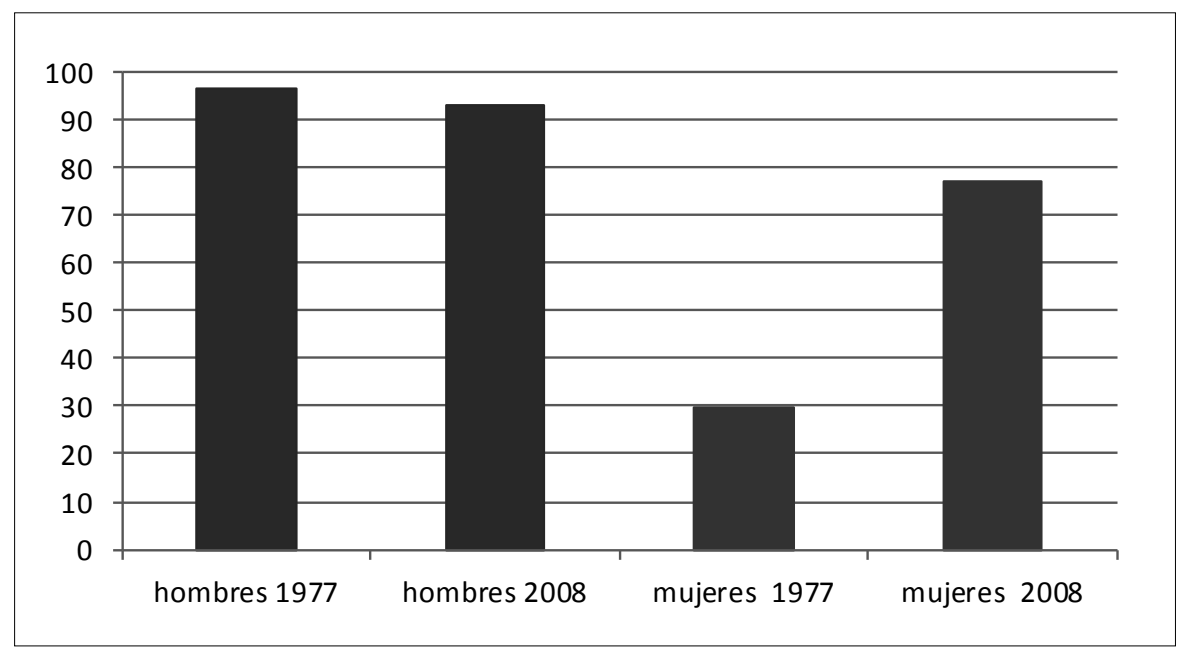

Fuente: Datos de la OIT. 
y menos al ocio. Propuestas como la de Carrasco et al. (2004) de una Encuesta de Población Activa no androcéntrica dan una imagen muy distinta del mundo del trabajo y su reparto según sexo, al incluir el trabajo doméstico y de cuidado, el estudio y el voluntariado, además del empleo.

El trabajo remunerado de forma directa, frecuentemente asociado a lo productivo, ha sido una categoría privilegiada del análisis económico y social, mientras que el otro trabajo ha permanecido durante largo tiempo en la penumbra analítica como asunto más propio de la biología o bajo la etiqueta de la reproducción, siempre supeditada a la producción. La incorporación entusiasta de las mujeres al mundo del empleo no va en paralelo al acceso de los hombres al mundo del cuidado. Esta constatación que, desde la perspectiva del sentido común dominante, parece obvia, no puede, sin embargo, dejar satisfechos a los sociólogos; más aún cuando las encuestas registran que, entre lo que se dice desear (compartir la responsabilidad de proveer al sustento familiar y la de cuidar de sus miembros) y lo que se hace, hay una distancia especialmente notoria en nuestro país (Consejo Superior de Investigaciones Científicas 2003; Centro de Investigaciones Sociológicas 2004:1).

\section{Gráfico 3.}

Porcentaje de tiempo que dedica a hogar y familia y tiempo promedio dedicado España 2003 y 2009

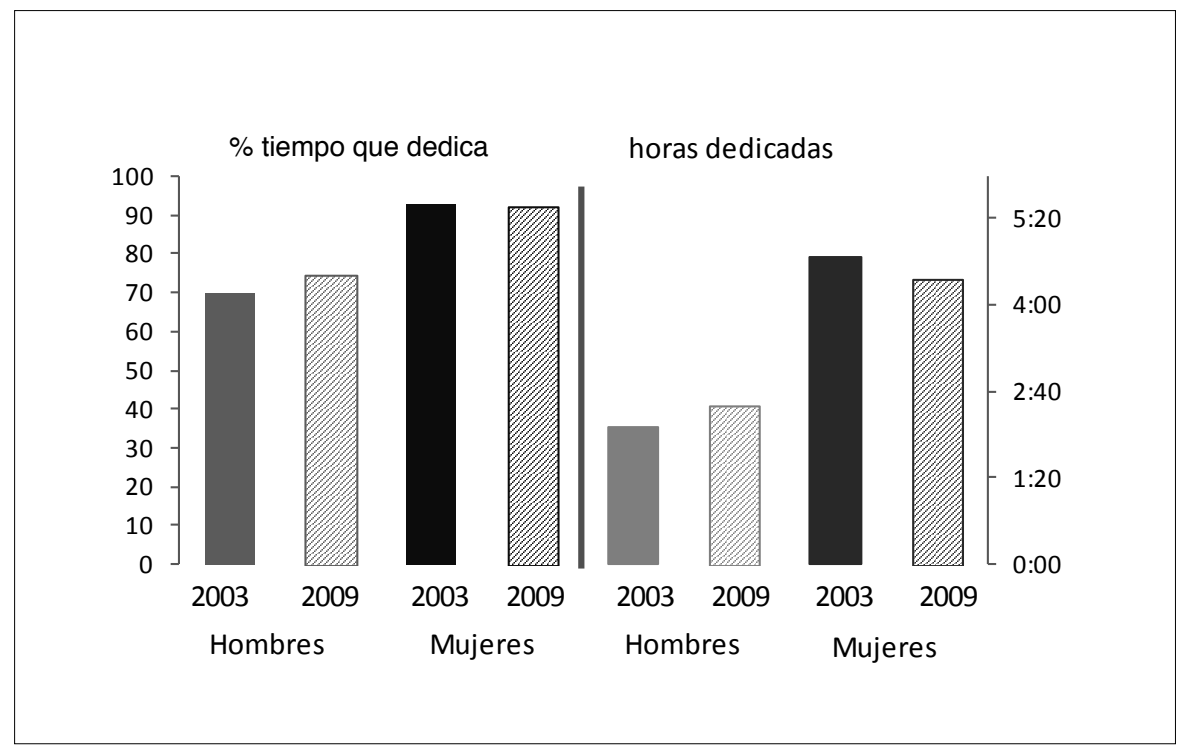

Fuente: Datos INE: Encuesta uso del tiempo 2009. 
Se percibe a las mujeres como ganadoras, al acceder al mundo del trabajo remunerado, en términos de autonomía personal y realización de sus potencialidades; pero también los hombres tienen en este proceso algo que ganar, aunque escasamente lo digan, al aminorarse la presión sobre su rol de proveedores y posibilitar el desarrollo de capacidades hasta ahora escasamente activadas; por ejemplo, la nueva forma de relación con los hijos que crea su cuidado directo. Para que los hombres también ganen en este proceso de cambio deben desearlo, a la vez que el entorno económico, social y político debe ser favorable; de no serlo hay otros futuros posibles, mucho menos deseables. Por ejemplo, uno de los efectos del modelo de proveedor universal (Fraser 1997), en el que todos los adultos de uno y otro sexo están en el mercado de trabajo, es que desaparece el salario familiar; es decir, la remuneración del trabajador masculino que se entendía debía cubrir las necesidades de las mujeres y los hijos. La tendencia a la individualización del salario sin la contrapartida de la reducción de la jornada laboral y sin, tampoco, la generalización de servicios y políticas sociales empeora las condiciones de vida, bien de las mujeres que siguen cuidando, como ocurre hoy, bien de las personas que no pueden valerse por sí mismas y no reciben el cuidado que necesitan o, bién, de los hombres, en la medida que ellos también cuiden.

\section{Gráfico 4.}

Distribución de algunas actividades en un día promedio

(sobre 7 días de la semana) España 2009.

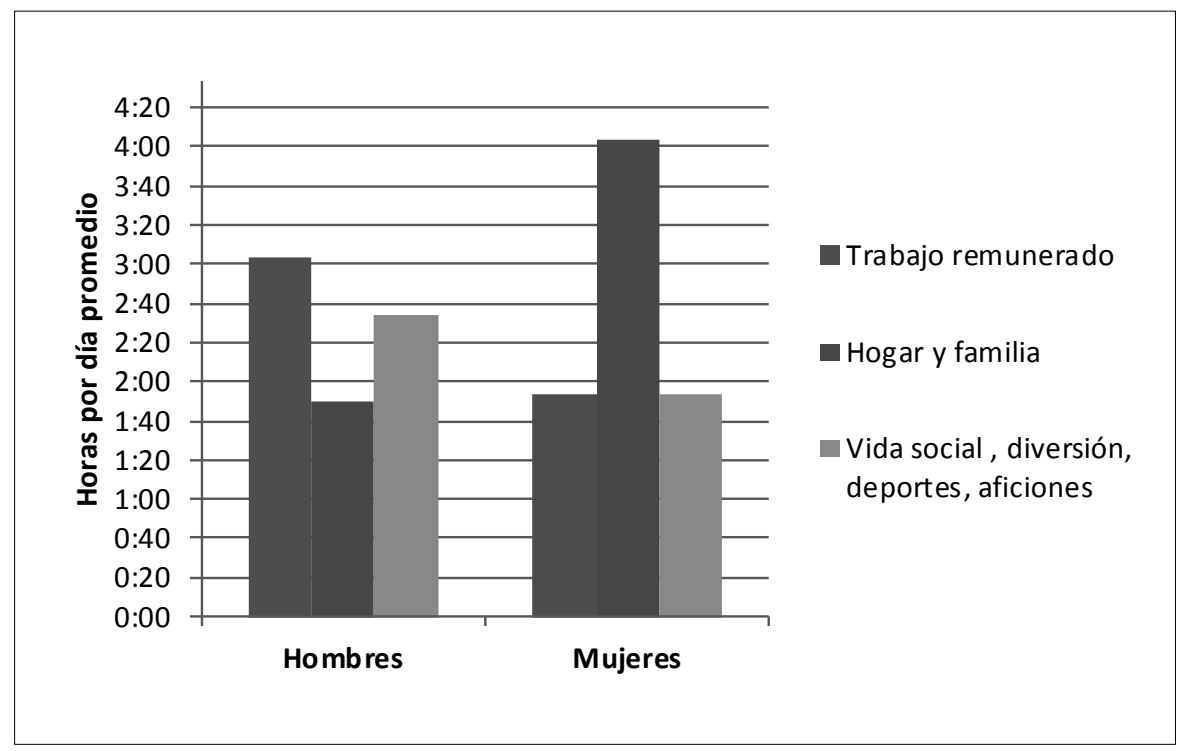

Fuente: Datos INE: Encuesta uso del tiempo 2009. 
El cuidado está fuertemente ligado a la identidad de género. En una argumentación circular se ha dado por supuesto que las cuidadoras lo son por ser mujeres, al tiempo que son mujeres porque cuidan. La masculinidad, por el contrario, se construye como la negación de la feminidad (Maccoby 1998; Connell 2000): no ser, no actuar como las mujeres y, por tanto, no cuidar. La teoría feminista, en especial la que se conoce como la "segunda ola", ha puesto en cuestión a través del concepto de "género" (Beltrán y Maquieira 2001) los determinantes biológicos de los roles sociales atribuidos a las mujeres; mientras que el feminismo cultural o de la diferencia ha reivindicado la aportación de las mujeres a la sociedad mediante el cuidado, tarea necesaria y compleja. Una vez que el empleo adquiere carácter universal —todos los adultos, hombres y mujeres están en el mercado de trabajo- la cuestión pendiente es la simétrica asunción de responsabilidades de los hombres en lo que se refiere al cuidado de las personas, en la que no se observan, más que de forma incipiente, nuevas prácticas sociales. Si las mujeres tuvieron que luchar por ocupar un espacio en el mundo público y laboral, encontrando numerosos obstáculos en su camino, a los hombres, por el contrario, se les anima y se les empuja a entrar en sus casas donde sin duda les esperan muchas sorpresas agradables, a pesar de la reticencia con la que empiezan a relacionarse de otra manera con el mundo que les rodea. Frente a la culpabilización o la inercia, quizá sea más fructífera la reflexión acerca de las dificultades que los hombres encuentran para cambiar, tanto en el nivel del saber hacer, como de su identidad e imagen social.

Este artículo pretende indagar en la relación entre el cuidado y la identidad de género tomando como hilo argumental la siguiente pregunta: ¿pueden los hombres cuidar? La primera parte aborda el concepto de cuidado y su progresiva expansión, de la maternidad, a la teoría del cuidado social. La segunda parte se centra en los procesos de construcción de la identidad de género, desde la doble perspectiva de la sociología y la teoría feminista. A continuación abordaré la discusión acerca de la noción de "cuidador universal" de Fraser y los interrogantes que ello abre para la propia noción de género, para concluir con la problematización de tal concepto como categoría binaria y la apertura a nuevos enfoques a los que no es ajena la cuestión del cuidado.

\section{Del CUIDADO MATERnal AL CUIDADO SOCIAL}

Desde sus orígenes, la sociología se ha interesado por la familia e incluso por la mujer, desde el positivismo de Comte (1980) y Durkheim ([1893] 1982), ([1897]1998) al funcionalismo de Parsons y Bales (1956), pero siempre en relación con su preocupación principal que era la cohesión social y el orden. Sólo algunos científicos sociales, que no se consideraban a sí mismos sociólogos, abordaron la desigualdad entre mujeres y hombres como un problema de la sociedad, desde distintas perspectivas -casi opuestas- como el liberalismo consecuente de Stuart Mill y Harriet Taylor ([1869] 2008; 2001) o las teorías de Marx y, en especial, Engels ([1884] 1982). No ha habido, sin embargo, 
desde la sociología una reflexión sistemática acerca de lo que ha aportado a lo largo de la historia el trabajo de las mujeres para hacer posible la supervivencia de las personas, o sobre la especificidad de la cultura femenina, con la excepción pionera de Simmel en los años veinte del pasado siglo ([1921] 1988), que no tuvo, sin embargo, mayor continuidad en la sociología. El concepto de "cuidado"1 ha sido elaborado por pensadoras feministas, como Delphy, Chodorow, Gilligan, Noddings o Daly y Lewis entre otras, e incorporado ya a la sociología del género y a la sociología de la familia como herramienta analítica de uso habitual.

La aportación de Delphy (1987) se puede considerar un antecedente del concepto de cuidado al señalar el valor económico de lo que denomina "modo de producción doméstico" en analogía con el "modo de producción capitalista". Desde una perspectiva psicoanalítica, Chodorow (1978) analiza el papel que desempeña la relación maternal en la formación de las identidades de género y la reproducción de la familia patriarcal. Replantea el enfoque freudiano del triángulo edípico basado en la idea del distanciamiento de la madre tanto del niño como de la niña, aunque de forma distinta en uno y otro caso. En el caso de las niñas, se añade la conciencia de la carencia, de la imposibilidad de ser como el hombre, la envidia del pene, para, finalmente, acabar aceptándose como seres incompletos. Chodorow, por el contrario, considera que en el caso de las niñas el desarrollo de la personalidad no es traumático, como en el de los niños, sino que hay una continuidad entre el rol que observan en la madre y el que ellas mismas como mujeres realizarán en el futuro. La madre es el espejo en el que se construye la identidad femenina sin necesidad de ruptura. Ello determina una predisposición a la empatía hacia las personas en la que se basa su capacidad para el cuidado. Los demás se perciben como semejantes con los que fácilmente se entablan relaciones de afecto y proximidad. Por el contrario, los niños tienen que romper el vínculo afectivo con la madre para llegar a ser hombres, lo cual determina el tipo de relaciones con las demás personas, marcadas por la distancia y la agresividad.

A partir del enfoque psicológico de Chodorow y de diversos estudios empíricos, Gilligan (1985) elabora la teoría del diferente desarrollo moral de las mujeres y los hombres que explicaría la "ética del cuidado" como característica del comportamiento de las mujeres. Se trata de un modelo ético basado en la responsabilidad hacia los demás que subyace a la actitud femenina, generalmente receptiva hacia las necesidades de las personas con las que se relacionan. El modelo moral masculino se basa más bien, según Gilligan, en la noción de "derechos" del individuo, de uno mismo, frente a una hipotética justicia imparcial. La competitividad y el egoísmo, así como la agresividad, son elementos constitutivos de la ética masculina. Frente a la idea de un desarrollo moral femenino

\footnotetext{
${ }^{1}$ Se ha impuesto ya en castellano la utilización del término "cuidado" para traducir del inglés la palabra "care", aunque no recoge toda su riqueza semántica ya que el uso de preposiciones como "for", "of" o "about" aporta toda una serie de matices que en nuestra lengua se pierden, sobre todo los relacionados con la implicación subjetiva de quien cuida en la tarea de cuidar.
} 
más simple o primario, Gilligan, en cambio, reivindica una ética basada en el cuidado de las personas y en la no violencia. Otras autoras como Ruddick (1989) o Noddings (1984) han proseguido la reflexión sobre la ética del cuidado como característica del comportamiento femenino, rechazando en todos los casos posibles interpretaciones de tipo biológico o esencialista. Si las mujeres tienen una especial predisposición para el cuidado, así como una actitud moral propia, ello es el resultado de la socialización femenina desde la primera infancia, el contexto de relaciones que entablan y las expectativas que hacia ellas se generan. Por tanto no son todas las mujeres las que desarrollan tal disposición al cuidado, ni tampoco se excluyen necesariamente los varones de la ética del cuidado.

Lo que el feminismo de la igualdad señalaba como causa primordial de la subordinación de las mujeres - la dedicación al mundo doméstico y al cuidado de las personasse reivindica desde el feminismo de la diferencia como actitud y prácticas femeninas necesarias para la supervivencia y la vida social. Ello no significa, sin embargo, que sea una vuelta a viejos planteamientos biologicistas o tradicionales sobre la división de roles de género, entre otras cosas por la elaboración teórica del cuidado como ámbito, hasta ahora invisible, de la realidad social. La cuantificación de su importancia arroja cifras para los años noventa que se sitúan entre un tercio y casi la mitad del PIB de países como Canadá (34\%), Noruega (38\%), Suiza (41\%) o Reino Unido (44\%) (Durán 2006:105). Las estimaciones del Instituto Nacional de Estadística son más modestas, 27,4\% (Instituto Nacional de Estadística 2008) aunque hay cálculos para comunidades autónomas que registran valores más elevados como Cataluña (40\%) (Carrasco y Serrano 2007) o Madrid (54,7\%) (Durán 2006).

Más allá de la maternidad, el cuidado se extiende a otras personas que no pueden valerse por sí mismas por su edad avanzada, la discapacidad o la enfermedad, y se extiende también a otros cuidadores que lo son por razón de parentesco en el ámbito de la familia (hijas, cónyuges y otros) o a cambio de una remuneración económica (asistentas, canguros, "fijas", "internas", "au pairs", etc.). Tanto en uno como en otro caso quienes cuidan son en la gran mayoría de los casos mujeres, seleccionadas según lógicas diversas que señalan a quién le corresponde hacerlo. Por ejemplo, suelen ser las esposas quienes cuidan al hombre que no puede valerse por sí mismo ( $41 \%$ de los casos) y en segundo lugar, las hijas (23\%). En cambio, en el caso de las mujeres que necesitan ayuda la cuidadora principal es en el $44 \%$ de los casos la hija, mientras el marido solo lo es en el 15\% (Tobío, Agulló Tomás, Gómez y Martín Palomo 2010:123). Se extiende también el ámbito del cuidado más allá de lo micro interpersonal al nivel macro en el que se integran, además de la familia, el Estado a través de las políticas sociales, el mercado y, aunque todavía escasamente desarrollado en nuestro país, el voluntariado. Daly y Lewis $(1998 ; 2000)$ han sistematizado el modelo de cuidado social incluyendo los diversos actores y agentes, así como la pluralidad de instituciones, prácticas sociales, normas y valores tanto implícitos como explícitos que lo conforman. La provisión de cuidados hoy en los países europeos y occidentales resulta de la combinación de recursos que aportan la familia, el Estado, el mercado y el voluntariado. Cuando se parte 
de la organización social del cuidado de las personas, la visión del bienestar difiere de los enfoques clásicos centrados en la educación, la sanidad o las pensiones. Por ejemplo, Francia y Alemania, generalmente adscritas al modelo conservador o corporativo (Esping Andersen 1993; 2000), aparecen desde la perspectiva del cuidado como casos distintos, a pesar de que coinciden en las generosas políticas sociales estatales y el protagonismo, en los últimos años muy matizado por tendencias universalistas, de la actividad laboral para la percepción de ayudas. En Alemania son las madres quienes suelen hacerse cargo directamente del cuidado de los menores de tres años, recibiendo por ello una compensación por parte del Estado. Por el contrario, en Francia hay una diversidad de alternativas (escuelas infantiles, cuidadoras en su propio domicilio ${ }^{2} 0$ trabajadoras domésticas) apoyadas por recursos públicos que explican la elevada participación laboral de las madres en este país. En otros casos, como los países del Sur de Europa, la familia sigue siendo el recurso fundamental para el cuidado de las personas a pesar del rápido aumento de las mujeres activas, especialmente en Portugal, donde la tasa de actividad laboral ${ }^{3}$ es del $68,9 \%$, más alta que la de Francia $(65,9 \%)$, y también en España donde alcanza el 63,2\% (Eurostat 2009:22).

\section{LA CONSTRUCCIÓN SOCIAL DEL GÉNERO}

Fue en los años cuarenta del pasado siglo cuando Simone de Beauvoir ([1949] 1998) rompe con una sola y breve frase el determinismo que ataba a las mujeres a la biología: "Una mujer no nace, se hace". Se abre así un nuevo camino en la reflexión acerca de la desigualdad entre mujeres y hombres, tras años de relativo estancamiento una vez alcanzado el derecho al voto femenino en los países occidentales después de la larga lucha de las sufragistas. Beauvoir se adelanta al construccionismo sociológico de Goffman o Garfinkel que indagarán en los mecanismos prácticos a través de los cuales se conforman las diferentes identidades de género de mujeres y hombres. Goffman (1977; 1979) parte de la idea de que lo sorprendente es que las pequeñas diferencias físicas entre hombres y mujeres se acrecienten tan grandemente en la percepción social, y afirma que sería posible en cualquier sociedad que se consideraran irrelevantes, haciéndose invisibles. Lo que analiza Goffman es la manera cómo tales pequeñas diferencias se convierten en grandes diferencias, así percibidas y generalmente aceptadas 4 . Desde las

\footnotetext{
${ }^{2}$ En francés, assistentes maternelles. Son cuidadoras que reciben en su casa a un número reducido de niños pequeños de cuyo cuidado se encargan mientras los padres y madres están trabajando. La Administración Pública asume tareas de control y supervisión de su trabajo.

${ }^{3}$ Datos para el año 2008 y una población de 15-64 años.

${ }^{4}$ Una línea argumental similar es la desarrollada por Gayle Rubin (1975:179) para quien, desde el punto de vista de la naturaleza, las mujeres y los hombres entre sí se parecen más que a cualquier otro ser vivo 0 cosa; o por Mary Jo Kane (1995) cuando afirma que las diferencias dentro de cada sexo en lo que se refiere
} 
diferencias en la forma de vestir, hablar y gesticular, hasta la segregación de espacios laborales, deportivos o la norma social de que los hombres deben ser más altos que las mujeres, existen toda una serie de mecanismos encaminados a construir la percepción de la diferencia entre ambos sexos como evidente. Analiza, entre otros ejemplos, el de los aseos públicos separados por sexos que genera cotidianamente la impresión de la relevancia de la distinción biológica, a pesar de que ninguna razón funcional en el uso que se hace de los mismos lo exige, como muestra el hecho de que en los domicilios particulares no se da tal separación, ni tampoco en ciertos tipos de lugares públicos, ni es tampoco una costumbre universal. Todo ello se refuerza con las imágenes que transmiten los medios de comunicación de lo que son y deben ser hombres y mujeres.

Los trabajos de Garfinkel (1984) también ofrecen interés para el estudio de la construcción social de la identidad de género, aunque su propósito no era tanto este como la constatación de que el conocimiento de sentido común, aquel con el que se desenvuelven en la vida cotidiana los participantes de una situación, es un producto de la interacción social. En realidad, eligió la identidad sexual como esquema dicotómico socialmente aceptado que hace imposible "pasar" de un lado a otro, de lo masculino a lo femenino, para demostrar que también algo aparentemente tan indiscutible como el hecho de ser hombre o mujer es el resultado de las convenciones aceptadas en la interacción cotidiana a través del sentido común. Garfinkel analizó pormenorizadamente el caso de Agnes, una joven de diecinueve años y aspecto femenino pero con genitales masculinos, socializada hasta la adolescencia como un varón. El estudio detallado del complejo proceso de aprendizaje de la condición femenina por parte de Agnes pone de manifiesto la omnipresencia del estatus sexual en la vida cotidiana y la variedad de situaciones en que está presente, aunque no se perciba explícitamente porque se da por supuesto, lo cual hace que el proceso de adquisición de una identidad femenina, cuando no ocurre de forma "natural" desde la primera infancia, revista una enorme dificultad y perviva un perpetuo temor a ser "descubierta", a pesar de que la operación de transformación se haya culminado con éxito, como en este caso.

El concepto de género, en la acepción todavía no asumida por la Real Academia de la Lengua Española ${ }^{5}$, está ya implícito en la obra de Beauvoir, aunque no utiliza este término, como diferenciación entre la construcción sociocultural de las características, comportamientos y actitudes de mujeres y hombres, que se superponen a los rasgos biológicos que les diferencian. El uso del género en este sentido no se generalizará hasta los años setenta del pasado siglo, en oposición —que no sustitución - al término

a capacidad deportiva son mucho mayores que las diferencias medias entre ambos sexos, es decir, hay muchas mujeres más fuertes y más rápidas que muchos hombres.

${ }^{5}$ Veáse el Informe de la Real Academia Española sobre la expresión "violencia de género" donde se justifica el rechazo del término. Consulta en línea 7/4/2010: http:/www.rae.es/rae/gestores/gespub000001.nsf/ (voAnexos)/archBB81F7452A4355C0C12571F000438E7A/\$FILE/Violenciadeg\%C3\%A9nero.htm, al que Chusa Lamarca Lapuente (2004) aporta una respuesta lingüística de interés. 
sexo reservado para la diferencia anatómica entre uno y otro. Kate Millet ([1969] 1995) es una de las primeras autoras que utiliza el concepto de género entendido como roles sociales que las mujeres aprenden desde la infancia, conformando una estructura de la personalidad que la sociedad considera acorde con su sexo. Se caracterizan por estar infravalorados respecto de los roles masculinos, lo cual constituye según la autora el elemento clave del patriarcado como sistema general de dominación de los hombres sobre las mujeres. La importancia del concepto de género estriba en la superación de las teorías biologicistas o esencialistas que hacen de la naturaleza del ser humano causa de la desigualdad entre mujeres y hombres, deslegitimando, así, el argumento de la imposible igualdad.

Gayle Rubin (1975) es otra de las autoras que contribuye a afianzar el concepto de "género" y diferenciarlo del "sexo". Se refiere al "sistema sexo-género" como las formas a través de las cuales la anatomía sexual se convierte en un sistema de desigualdad basado en prohibiciones para las mujeres y derechos exclusivos para los hombres. Chafetz (1988) plantea que el género impregna todas las relaciones, procesos e instituciones sociales, por lo que constituye el concepto central de la teoría feminista. La tarea de la investigación con perspectiva de género es desvelar el carácter "generizado6" de la sociedad.

La identidad de género es uno de los componentes del género, entre otros como las atribuciones, las ideologías, los símbolos, las normas o las instituciones (Beltrán y Maquieira 2001:167-172). La identidad se construye a lo largo de toda la vida a partir de las definiciones sociales y de las autodefiniciones que la persona va elaborando como hombre o mujer. Tiene un doble carácter de identificación con modelos y de rechazo a lo que no es conforme a la identidad en construcción. Todo ello crea un sistema de distinciones entre géneros y de percepción de semejanzas dentro de cada género.

La identidad femenina ha sido largamente estudiada directa e indirectamente por las pensadoras feministas y las ciencias sociales en general. El segundo sexo de Simone de Beauvoir (1998) puede entenderse así, como una reflexión sobre la "otra", como la identidad marcada por la inmanencia. O la obra de Betty Friedan como la identidad que resulta del encierro doméstico. Y gran parte de las aportaciones del feminismo de la diferencia (Irigaray 1978, 1992); Cixous 1983; Chodorow 1978; Gilligan 1985) abordan la cuestión de la identidad femenina, no sin que deje de estar presente el dilema igualdad-diferencia ya apuntado por Mary Wollstonecraft en el siglo XVIII, tal como señala Pateman (2000). Los dos caminos posibles para la liberación de las mujeres resultan no serlo bajo el dominio patriarcal. Adentrarse en el camino de la igualdad y en el mundo de lo público como ciudadanas de pleno derecho, como los hombres, supone olvidar la diferencia que entraña encargarse de los hijos y de los mayores; es decir, olvidar que alguien — pero no necesariamente siempre las mujeres— debe cuidar a quienes lo necesitan. Por otro lado, reivindicar el apoyo de la sociedad por el hecho de cuidar supone

\footnotetext{
${ }^{6}$ En inglés, gendered, neologismo que no tiene una fácil traducción.
} 
reproducir la desigualdad y permanecer como eternas cuidadoras. Bajo la dominación patriarcal, concluye Pateman, el dilema no tiene solución.

Mucho menos estudiada ha sido la identidad de género masculina. La asimilación del "hombre" a lo humano, a lo universal o al poder ha hecho que el proceso a través del cual un individuo de sexo masculino adquiere el género correspondiente haya sido dado por supuesto. El desarrollo de la investigación sobre los hombres ${ }^{7}$ o sobre las masculinidades ${ }^{8}$ desde una perspectiva de género es relativamente reciente, aunque se dispone ya de obras de recopilación que dan cuenta del estado de la cuestión en este campo de estudio (Kimmel, Hearn y Connell 2005; Adams y Savran 2002). Incluso en España la producción de los últimos años permitió en el último Congreso de la Federación Española de Sociología incluir en el volumen recopilatorio de la sociología española reciente (Pérez Yruela 2007) un capítulo sobre lo realizado en este campo en nuestro país (Guasch 2007).

Los trabajos sobre la construcción de la identidad de género masculina señalan dos grandes temas. Por un lado, la distinción o la oposición a todo lo femenino. Un hombre es aquello que no es una mujer (Maccoby 1998). Cuando un bebé llega al mundo lo primero que las personas preguntan y los progenitores aclaran es su sexo, lo cual orienta lo que se dice y como se le trata. Desde muy temprana edad los varones forman grupos de pares del mismo sexo e incorporan el mandato social de la proscripción del comportamiento femenino, lo cual marca la relación con la madre, con las niñas y en general con lo que perciben como el mundo de las mujeres, lo doméstico y el cuidado de las personas. Los niños se alejan de los adultos antes y en mayor medida que las niñas, lo que se les permite, aunque, o precisamente porque, reproducen lo que, explícita o implícitamente, la sociedad espera de ellos como futuros hombres en el sentido "generizado" del término. El rechazo que produce en sus pares todo lo que se asimile a lo femenino es uno de los elementos clave en el proceso de conversión de un niño en un hombre. Por otra parte, los padres (hombres) tienden a reforzar los estereotipos de género, especialmente en sus hijos varones (Fagot y Leinbach 1993), al tiempo que paradójicamente la identidad masculina se considera más frágil que la femenina (Mead 1949; Chodorow 1978; Adams y Coltrane 2005).

El segundo elemento repetido en la investigación sobre la formación y las características de la identidad masculina se refiere a la competitividad y la agresividad. Simone de Beauvoir (1998) la interpreta como la asunción del riesgo de la muerte asociada a la trascendencia frente a la inmanencia en la que permanecen recluidas las mujeres. Kaufman (1998), por ejemplo, señala que los hombres construyen su masculinidad en torno a una triple violencia: contra las mujeres, contra otros hombres y contra sí mismos. Ello se manifiesta en los juegos infantiles y de manera especial en la práctica deportiva como aprendizaje del dolor y el esfuerzo, así como la fuerza y el

\footnotetext{
${ }^{7}$ En inglés, "Men's Studies"

${ }^{8}$ En inglés, "Masculinities"
} 
dominio sobre las mujeres (Messner 2005). Se reproducen así en la cotidianeidad más prosaica los ritos iniciáticos de la masculinidad de los que nos hablan la mitología y la antropología. La mayor parte de los actos violentos son cometidos por hombres en una proporción que se ha estimado de diez a uno (Bowker 1998) lo cual no significa que todos los hombres lo sean o que ninguna mujer los cometa. Los hombres, además, son también víctimas de sus conductas de riesgo, tal como revelan los datos de mortalidad por accidentes, suicidios, drogas o alcohol mucho más elevados que los de las mujeres (Castells y Subirats 2007). Todo ello conduce a Jeff Hearn (1990:85) a afirmar que el problema no son los hombres violentos sino la propia "masculinidad normal". El cambio que hoy protagonizan las mujeres, sin embargo, transforma las bases patriarcales de la sociedad. Se puede afirmar que vivimos ya una fase de transición que los hombres perciben frecuentemente como desorientación tal como han puesto de manifiesto, entre otros, Gil Calvo (1997) o Flaquer (1999). El viejo patriarca se resquebraja, ¿emerge un nuevo hombre?

\section{¿POR QUÉ NO CUIDAN LOS HOMBRES?}

Habría que responder, en primer lugar, que ello no es del todo cierto: hay hombres que cuidan aunque sean una minoría. En mayor o menor medida, de mejor o peor grado, pero existen. Cuidan, por ejemplo, los padres de sus hijos, pero en menor medida que las madres, incluso si éstas trabajan. Según datos de la Encuesta Consejo Superior de Investigaciones Científicas sobre Usos del Tiempo, la dedicación semanal al cuidado de hijos pequeños por los hombres es algo menos que la tercera parte de la de las mujeres (3,03 horas frente a 10,78 horas). Lo que más llama la atención es que no incremente de forma clara durante los fines de semana cuando normalmente no trabajan (Alberdi y Escario 2007). Según datos de la Encuesta de Redes Familiares de Andalucía de 2006, el 31,6\% de los hombres con hijos menores les dedica al menos 40 horas semanales, porcentaje que se rebaja al $25,6 \%$ cuando son sus cónyuges quienes responden a la pregunta. En el caso de las mujeres, el 78,9\% afirma dedicar al menos tal número de horas, porcentaje que también rebajan sus parejas, en este caso al $71,9 \%$. La dedicación es especialmente intensa entre las más jóvenes, teniendo una menor incidencia la edad entre los hombres (Rodríguez Pascual 2008:32). Esta misma fuente nos da más información acerca de las tareas que los hombres realizan en mayor medida9: cuidar en vacaciones $(86,4 \%)$, juegos y bricolage $(72,4 \%)$, pasear e ir al parque $(69,8 \%)$, cuidar en circunstancias extraordinarias $(68,8 \%)$, turismo y excursiones $(65,7 \%$ ) y cuidar a los menores en edad preescolar $(61 \%)$.

\footnotetext{
${ }^{9}$ La pregunta estaba formulada en términos dicotómicos, realizar o no realizar habitualmente, admitiendo hasta cinco personas que pudieran asumir la tarea, por lo que la respuesta puede ser sí aunque en realidad se haga en contadas ocasiones.
} 
Las actividades que una menor proporción de padres realiza con sus hijos son llevar a los niños al colegio o a la guardería (42,7\%), participar en las tutorías escolares $(45,9 \%) 0$ practicar deportes con ellos (44\%), preparar el desayuno (53,2\%) o encargarse del baño, del aseo y de cambiar los pañales (54,9\%). Difiere, por tanto, no solo la cantidad de tiempo dedicada a los hijos, sino también el tipo de actividades que los hombres hacen con ellos, más orientadas al juego y a lo extra-doméstico.

Otra forma de detectar el papel de los hombres en el cuidado de sus hijos pequeños es a través de los permisos laborales. Los datos son muy distintos en lo que se refiere a la parte del permiso de maternidad de la que pueden disfrutar (hasta diez de las dieciséis semanas) o el permiso parental (hasta tres años durante los tres primeros años de vida del menor) y el permiso de paternidad, quince días reservados exclusivamente a los hombres (Tobío, Agulló Tomás, Gómez y Martín Palomo 2010:167171). En los dos primeros casos, la proporción de hombres que decide disfrutarlos es bajísima y el incremento que se observa es muy lento. En 2007, solamente el 1,6\% de los padres varones tomó alguna parte del permiso de maternidad, lo cual supone un pequeño aumento en comparación con las cifras del año 2000, 0,98\%. En cuanto a las excedencias por cuidado de menores, son un número relativamente reducido de casos, pero son abrumadoramente femeninas $(95,7 \%)$. Por el contrario, son amplia mayoría, aproximadamente tres de cada cuatro, los que se acogen al permiso de paternidad.

Las diversas fuentes disponibles muestran un lento incremento de la implicación de los padres en el cuidado de sus hijos, aunque su participación está todavía muy lejos de la de las mujeres, incluso en países como Francia (Barrère Maurisson 1992) en los que la actividad laboral femenina cuenta con una larga tradición y es muy mayoritaria. Paradójicamente los hombres ingleses participan más en las tareas domésticas y de cuidado que los hombres en Francia, donde la gran mayoría de las mujeres está ocupada a tiempo completo desde hace décadas y hay amplias y variadas políticas sociales. En el Reino Unido, por el contrario, las políticas son más escasas, lo cual hace, tal como plantean Gregory y Windebank (2000), la actividad de los hombres en el hogar imprescindible, a pesar de que la ocupación femenina a tiempo parcial es alta. A veces los hombres cuidan, y seguramente lo han hecho siempre, porque no hay más remedio, como ocurre también entre hombres de clase baja como ha estudiado Gutmann (1996) en el caso mejicano, una realidad que podría permanecer oculta precisamente debido a los estereotipos de la masculinidad. Quizá algunos hombres cuidan más de lo que reconocen. Otros en cambio reivindican hoy la custodia de sus hijos en caso de separación, cuestión no exenta de polémica, y también hay hombres que se organizan para fomentar su rol de padres cuidadores (Plataforma por Permisos Iguales e Intransferibles de Nacimiento y Adopción 2009). El concepto de "paternidad sostenible" (Alberdi y Escario 2007) recoge la nueva realidad de un vínculo basado en la relación directa y próxima de los hombres con sus hijos, sin la intermediación de la madre y con independencia de la relación conyugal, es decir, que permanece - de hecho en ocasiones se acrecienta- después de una separación o divorcio de los padres. 
Cuidan, asimismo, los abuelos de sus nietos, tan frecuentemente como las abuelas, aunque con una dedicación menor y en tareas distintas (Tobío 2005:151-173; Tobío, Agulló Tomás, Gómez y Martín Palomo 2010:109-120; Ibarra y Pinazo 2008; Badenes y López 2009). A pesar del esfuerzo que ello supone, lo hacen con placer, encontrando, aunque sea tardíamente, un tipo de relación gratificante que no pudieron tener con sus propios hijos. Quizá el nuevo hombre esté representado mejor que nadie por los abuelos de hoy.

E incluso hay varones adultos que cuidan de mayores, generalmente, como se ha comentado antes, de sus cónyuges. Pero son pocos, y sabemos poco de ellos, porque suele ser más bien al revés. Una combinación de la biología y de las prácticas sociales hace que con más frecuencia sean las esposas quienes cuiden de sus maridos que no pueden valerse por sí mismos. La diferencia de esperanza de vida entre hombres y mujeres a favor de estas últimas, añadida a la norma social de la diferencia de edad a favor del hombre, hacen que normalmente quien necesita cuidado antes sea él y quien esté disponible sea ella. ¿Una estrategia patriarcal inmemorial?

No sólo se cuida por parentesco, sino también como actividad laboral. Y lo mismo ocurre en el caso de los hombres, aunque sea todavía una ocupación minoritaria. Sobre todo cuidan a mayores tanto en marcos institucionales como en familias, generalmente a hombres que no pueden valerse por sí mismos. También cuidan a menores de ambos sexos en guarderías o escuelas infantiles. Son todavía pocos y no deja de haber algunos recelos hacia ellos en lo que respecta a la intimidad que supone el cuidar (Brannen y Moss 2003).

En resumen, hay hombres que cuidan, por tanto no es imposible que lo hagan, pero son minoría y lo hacen todavía como algo excepcional o novedoso. ¿Qué obstáculos hay para que los hombres cuiden habitualmente, ahora que está plenamente normalizado que las mujeres estén ocupadas en un empleo? Podrían señalarse al menos tres: saber, poder y querer. Cuidar requiere una amplia variedad de conocimientos prácticos, distintos, además, según quién sea la persona necesitada de cuidados. Desde el aseo y la higiene de quien no puede hacerlo por sí mismo hasta la alimentación o la movilidad, pasando por el bienestar físico y psíquico. Son conocimientos que, como todos, requieren aprendizaje; aunque hasta ahora se han transmitido informalmente entre mujeres 0 , frecuentemente, han tenido que adquirirlos en la práctica ante la urgencia de la demanda de ayuda. No están inscritos, sin embargo, en la genética femenina ni hay nada, excepto la temprana socialización, que haga a las mujeres más dotadas para estas tareas que los hombres. La resistencia a aprender es, en ocasiones, una estrategia masculina para entrar en el mundo del cuidado (Tobío 2005:209-214).

Muchas veces los hombres no pueden cuidar, fundamentalmente, porque tienen una elevada jornada laboral, 42,8 horas semanales de media, más que en el conjunto de los países de la UE (Eurostat 2009:32) y largos desplazamientos de la casa al trabajo, más largos que los de las mujeres (Tobío 2003). Tampoco tienen un entorno social que entienda que en ciertos momentos de la vida hay otras cosas más 
importantes que el empleo y decidan tomar una excedencia para el cuidado de hijos 0 una parte del permiso de maternidad. También ocurre que los necesitados de cuidado prefieren que sea una mujer, preferentemente de la familia quien lo haga. Es decir, a través de mecanismos diversos se les excluye del cuidado. Por ello, es tan importante el permiso de paternidad no transferible a la madre que en nuestro país como en otros (Lister et al. 2007; Ellingsaeter y Leira 2006), ha tenido un gran éxito. Da a los hombres la legitimidad que necesitan para ejercer como cuidadores. Entender el cuidado como un derecho que también tienen los hombres ayuda a que puedan experimentar esa parte de la vida, además de acceder a un ámbito laboral hasta ahora casi exclusivamente femenino.

La tercera razón por la que los hombres no cuidan normalmente es porque no quieren, lo cual tiene mucho que ver con la identidad de género. Porque no es un trabajo de hombres, no les corresponde, no se sienten aludidos ni interpelados. No es su responsabilidad, creen, y si lo es, con frecuencia encuentran la manera de transferirla a alguna mujer del entorno familiar 0 a una asalariada.

En realidad saber, poder y querer no son aspectos que se puedan diferenciar fácilmente sino que conforman juntos una cultura del cuidado y se refuerzan mutuamente, excluyendo de facto a los varones. Si no quieren es porque no saben 0 si no saben es porque no quieren o no pueden. $Y$ si no pueden es porque no quieren. Incorporar a los hombres al mundo del cuidado de las personas exige un nuevo contexto, un nuevo modelo social y familiar como el que Nancy Fraser (1997) ha denominado "Modelo de cuidador universal". Se contrapone a otros dos modelos posibles que suponen un avance en la igualdad de género, pero quedan atrapados en el dilema de Wollstonecraft, el "Modelo del proveedor universal" y el "Modelo de paridad del cuidador". El primero supone que todos los adultos, hombres y mujeres proveen económicamente a través del empleo remunerado. Se inscribe en la perspectiva del feminismo de la igualdad, pero deja pendiente la cuestión del cuidado. ¿Quién se encarga de los menores y los mayores? El segundo, responde más bien a la perspectiva del feminismo de la diferencia, en el sentido de igualdad en la complementariedad. Asegura que quienes cuidan, previsiblemente las mujeres, no se ven penalizados por el hecho de hacerlo en sus derechos económicos presentes y futuros. Reconoce, por tanto, la importancia del cuidado pero reproduce roles de género diferentes. El tercer modelo, también llamado de integración, presupone que todos los empleos tienen en cuenta que los trabajadores de ambos sexos tienen responsabilidades de cuidado a las que deben atender. Ello supone, entre otras cosas, que la jornada laboral a tiempo completo debe ser más corta para todos, sin que haya como ahora, explícita o implícitamente, empleos de mujeres, a tiempo parcial o limitado, y de hombres, caracterizados por la disponibilidad sin límites para el tiempo laboral. Una parte del trabajo de cuidado se haría en instituciones, pero otra, especialmente en el caso de los menores, correría a cargo de la familia nuclear o extensa. Incluso, plantea Fraser, aquellas personas dependientes en su familia podrían ayudar a quienes lo necesiten, y ser a su vez ayudadas cuando lo requirieran. 
Este tercer modelo integra adecuadamente la igualdad de género, la actividad laboral y el cuidado, exigiendo una reestructuración en profundidad del mundo doméstico y de la propia familia. Es coherente, además, con el modelo que dicen los españoles y los europeos desear. Permanece, sin embargo, el reto de cómo hacer compatibles el derecho a ser cuidado, el derecho a cuidar y el derecho a no cuidar, hasta ahora privativo de los hombres (Leira 2002).

\section{LA CRÍTICA DE LA CONCEPCIÓN DICOTÓMICA DEL SEXO Y DEL GÉNERO}

El cuestionamiento de la diferenciación de roles entre hombres y mujeres se refuerza con la aparición de enfoques que critican la oposición radical entre lo masculino y lo femenino. Ello se inscribe en la problematización de las categorías dicotómicas como soporte analítico del pensamiento científico, característico de algunas corrientes de la teoría feminista. La antropóloga Michelle Rosaldo (1983), por ejemplo, argumenta que la dicotomía entre el mundo doméstico y público, así como su adscripción respectiva a las mujeres y a los hombres, es una herencia de la teoría social decimonónica que impone un deber ser a procesos sociales mucho más complejos. Otras pensadoras como Sacks (1979) o Nelson (1974) han discutido la relación doméstico-público, mostrando como las fronteras entre uno y otro son fluidas, y en algunas sociedades inexistentes. En el ámbito de lo personal, frente a la concepción binaria dominante se concibe un gradiente en el que caben opciones intermedias o combinaciones, tanto en lo que se refiere a la anatomía como a la percepción o a las prácticas sexuales.

La idea del hombre y la mujer como únicas realidades se contrapone al hecho de una gradación biológica de los caracteres físicos femeninos y masculinos, así como a la existencia de individuos intersexuales, reconocidos en muchas culturas como un tercer sexo. Se han identificado al menos cinco sexos biológicos diferenciados (Fausto-Esterling, 1998), al añadir a los individuos con rasgos exclusivamente femeninos o masculinos aquellos que tienen ambos, en mayor o menor media. Son los herms, hermafroditas puros, ya reflejados desde la antigüedad en el arte, los merms y los ferms. Antes Kinsey et al [1948] 1998) habían definido la homosexualidad y la heterosexualidad como un continuum en una gradación de cero a seis, y Rubin (1984) señaló el carácter jerárquico de las prácticas sexuales desde la monogamia matrimonial, forma socialmente aceptada como legítima, hasta el travestismo, la transexualidad, el sadomasoquismo y la prostitución, en el extremo opuesto. Ha sido la denominada teoría queer la que ha hecho de la sexualidad no heterosexual objeto específico de análisis, a partir de la apertura a todo tipo de modalidades de auto-identificación y formas de relación en este ámbito (De Lauretis 1989; Osborne y Guasch 2003). La última fase en el proceso de de-construcción del sexo y del género apunta a una "proliferación" tanto desde la exclusión de lo que se rechaza como de su reivindicación desde estrategias de ruptura del orden sexual, tal como plantea Butler ([1990] 2001). Hasta qué punto ello resta capacidad analítica tanto a la categoría sexo como a la de género es materia de debate, así como su extensión en la 
percepción que de su identidad tienen las personas. Sí parece indudable, en cambio, la creciente diversificación de identidades, derivadas o no de rasgos biológicos, y la mayor creatividad individual en su construcción (Hearn 2002a y 2002b).

Todo ello sitúa en otros términos la cuestión del cuidado. No se trataría ya de estudiar hasta qué punto las mujeres siguen cuidando o en qué medida los hombres empiezan a hacerlo sino cómo organizar la atención a quienes no pueden valerse por sí mismos en un nuevo contexto en el que la identidad de género reduce su carácter determinante de los comportamientos. En este sentido el relajamiento, al menos, de las estrictas normas de género puede ser una oportunidad para fortalecer la noción de cuidado como derecho y obligación de las personas, individual y colectivamente, con independencia de la auto-percepción sexuada. Nuevas experiencias y realidades sociales como la de los matrimonios homosexuales con hijos permitirá en los próximos años saber más acerca de la superación o el mantenimiento de la diferenciación de roles más allá de la heterosexualidad.

\section{Conclusiones}

No hay nada en la biología femenina que determine la dedicación de las mujeres al cuidado, ni tampoco nada en la masculina que lo impida, tal como la diferenciación entre sexo y género pone de manifiesto. Es la normalidad social del patriarcado la que ha impuesto una división del trabajo en la que las mujeres cuidan y los hombres aportan el sustento familiar. No se trata de una mera división funcional, sino que el valor atribuido a unas y otras tareas difiere, ocupando el cuidado el menor rango. 0 , quizás, como Mead ([1935] 2006) o Rosaldo (1979) han señalado, sea aquello que hacen los hombres lo que recibe una mayor consideración social, con independencia de su contenido concreto.

Se investiga por qué las mujeres acceden al mundo laboral formalizado y al ámbito de lo público. ¿Por qué trabajan las mujeres? Es esta una pregunta de investigación cuyas respuestas son múltiples: de la necesidad económica familiar al deseo de independencia económica individual, de la autonomía personal a la aplicación de los conocimientos adquiridos o la profesión aprendida y de la relación social a la autorrealización (Tobío 2005). Más allá del por qué, los datos muestran que la actividad laboral de las mujeres es ya un hecho incontestable. Trabajan casi todas, a lo largo de toda la vida y en proporciones parecidas a las de los hombres, tal como se ha visto anteriormente. Es, además, un hecho reciente en nuestro país. Hace menos de treinta años la ocupación laboral era solo una de las opciones posibles para las mujeres y ni siquiera la mayoritaria. Lo sorprendente hoy es que a la vez siguen cuidando casi tanto como antes. Es decir, superponen los viejos roles femeninos a su nueva dedicación laboral. Inevitablemente las miradas se dirigen ahora a los hombres. Pero la pregunta no es simétrica a la que se hace acerca de las mujeres. No es por qué cuidan los hombres, sino por qué no lo hacen. A ello subyace la idea de que deberían hacerlo en lógica reciprocidad hacia el cambio protagonizado por las mujeres, que también les incumbe. 
Se abre así la puerta a medidas que animen a los hombres a entrar en ese mundo. La respuesta al porqué de la reticencia masculina hacia el cuidado no es fácil ni unívoca, como tampoco lo son las razones de las mujeres para trabajar. Conocer los variados obstáculos a los que se enfrentan los hombres para cuidar requiere mucha más investigación de la hasta ahora realizada. En este artículo se han explorado a modo de hipótesis algunas explicaciones posibles que puedan orientar futuros trabajos y ayudar a conocer un aspecto fundamental del cambio en las relaciones de género que hoy vivimos.

Se va abriendo la puerta a una pluralidad de situaciones y combinaciones para cada persona a lo largo de la vida. También los hombres pueden cuidar a tiempo completo durante ciertos periodos o incluso asumir esta dedicación como la principal, a la vez que las mujeres pueden centrarse en la actividad laboral. 0 , lo que parece más probable, compartir de forma flexible unas y otras tareas.

Sin embargo, la inercia del cuidado como cuestión de mujeres persiste a pesar de que éstas desempeñan una creciente actividad en el mundo de lo público y de que hay políticas específicas para promover la incorporación de los hombres al cuidado. A pesar, también, de que hay hombres que cuidan y, seguramente, los ha habido siempre. Como ha habido siempre mujeres que han desempeñado tareas productivas de todo tipo. La cuestión es qué factores explican esa inercia que separa a los hombres del cuidar cuando hay otros que presionan en sentido contrario tanto a nivel micro, las mujeres, como a nivel macro, algunas políticas sociales. Más aún, cuando la vieja responsabilidad masculina de la provisión de sustento para la familia ahora se aligera al compartirse.

Tal como se ha visto a lo largo de las páginas anteriores, hay tres grandes tipos de obstáculos que dificultan la dedicación de los hombres al cuidado relacionados con el saber, el poder y el querer. Cuidar requiere un aprendizaje que las generaciones de mujeres se han transmitido de manera informal, lo cual seguramente ha contribuido a la idea de su carácter innato. La incapacidad masculina para el cuidado no es con frecuencia más que la manifestación de una carencia de conocimientos mucho más complejos de lo que habitualmente se cree. Además, cuidar requiere poder hacerlo; es decir, un contexto social, laboral, personal y familiar que lo acepte y lo haga posible, lo cual no es habitual en el caso de los hombres. Finalmente, para cuidar hay que querer hacerlo, por las razones que sea, que pueden ser muy variadas. Ello es, probablemente, el aspecto clave que explica la reticencia masculina ya que tiene que ver con la identidad, con la idea, no necesariamente consciente, de lo que es un hombre y la resistencia de los modelos patriarcales que todavía impregnan nuestra sociedad. Existen nuevos modelos pero su capacidad de reconocimiento y aceptación no está ni mucho menos generalizada.

El cuestionamiento de la concepción binaria del sexo como oposición dicotómica determinista por parte de las corrientes más actuales de los estudios de género refuerza la concepción del cuidado como responsabilidad universal. Pierde peso como rasgo e indicador de diferencia sexual, pero lo gana como actividad necesaria y común a las personas, ellas y ellos, que a ambos atañe. Los hombres que cuidan serán distintos, pero sin duda mejores y más felices. 


\section{REFERENCIAS BibLIOGRÁFICAS}

Adams, M. y S. Coltrane. 2005. "Boys and Men in Familias". Pp. 230-248 en Handbook on Studies on Men \& Masculinities edited by M. S. Kimmel J. Hearn y R. W. Connell. Thousand Oaks/Londres/ Nueva Delhi: Sage Publications.

Adams, R. y D. Savran. 2002. The Masculinity Studies Reader. Malden Massachusetts: Blackwell Publishers.

Alberdi, I. y P. Escario. 2007. Los hombres jóvenes y la paternidad. Bilbao: Fundación BBVA.

Badenes, N. y M. T. López. 2009. "Doble dependencia: abuelos que cuidan nietos en España". Informe de investigación. Universidad Complutense.

Barrère-Maurisson, M. A. 1992. La division familiale du travail. La vie en double. París: Presses Universitaires de France.

De Beauvoir, S. [1949] 1998. El segundo sexo. Madrid: Cátedra, 2 vols.

Beltran, E. y V. Maquieira. 2001. Feminismos. Debates teóricos contemporáneos. Madrid: Alianza Editorial.

Brannen, J. y P. Moss. 2003. Rethinkings Children's Care. Buckingham: Open University Press.

Bowker, L. H. 1998. Masculinities and Violence. Thousand Oaks CA: Sage Publications.

Butler, J. [1990] 2001. El género en disputa. Barcelona: Paidós.

Carrasco, C. y M. Serrano. 2007. Compte satèllit de la producció domèstica (CSPD) de les llars de Catalunya 2001. Barcelona: Institut Català de les Dones.

Carrasco, C., M. Mayordomo., M. Domínguez y A. Alabart. 2004. Trabajo con mirada de mujer. Propuesta de una encuesta de población activa no androcéntrica. Madrid: Consejo Económico y Social.

Chafetz, J. 1988. Feminist Sociology: En Overview of Contemporary Theories. Itasca: Peacock.

Chodorow, N. 1978. The Reproduction of Mothering. Psychoanalysis and the Sociology of Gender. Berkeley: University of California Press.

CIS. 2004. "Estudio 2556: Barómetro de febrero", en Boletín 34. Madrid: CIS.

Cixous, H. 1983. "The Laugh of the Medusa". Pp. 196-202 enThe Signs Reader: Women, Gender and Scholarship. Chicago: University of Chicago Press.

Comte, A. [1844] 1980. Discurso sobre el espíritu positivo. Madrid: Alianza Editorial.

Connell, R. W. 2000. The Men and the Boys. Berkeley: University of California Press.

CSIC. 2003. Encuesta CSIC sobre uso del tiempo en España. Madrid: CSIC. 
Daly, M. y J. Lewis. 1998. "Introduction: conceptualising social care in the context of welfare state restructuring in Europe". Pp. 1-24 en Gender Social Care and Welfare State Restructuring in Europe, edited by J. Lewis. Ashgate: Aldershot.

Daly, M. y J. Lewis. 2000. "The concept of social care and the analysis of contemporary welfare states." British Journal of Sociology 51: 281-299.

De Lauretis, T. 1989. Technologies of Gender. Bloomington: Indiana University Press.

Delphy, C. 1987. "Modo de producción doméstico y feminismo materialista". Pp 17-28 en Mujeres: Ciencia y práctica política, editado por C. Amorós, L. Benería, C. Delphy, H. Rose y V. Stolcke. Madrid: Debate.

Durán, M. A. 2006. La cuenta satélite del trabajo no remunerado en la Comunidad de Madrid. Madrid: Comunidad de Madrid.

Durkheim, E. [1893] 1982. La división del trabajo social. Madrid: Akal.

Durkheim, E. [1897] 1998. El suicidio. Madrid: Akal.

Ellingsaeter, A. E. y A. Leira. 2006. Politicising Parenthood in Scandinavia. Gender Relations in Welfare States. Bristol: Policy Press.

Engels, F. [1884] 1982. El origen de la familia, la propiedad privada y el Estado. Madrid: Fundamentos.

Esping-Andersen, G. 1993. Los tres mundos del Estado del Bienestar. Valencia: Alfons el Magnànim.

Esping-Andersen, G. 2000. Fundamentos sociales de las economías postindustriales. Barcelona: Ariel.

Eurostat 2006. Harmonised European Time Survey. Luxemburgo: European Commission.

Eurostat 2009. Labour Market Statistics. Luxemburgo: European Commission.

Fausto-Esterling, A. 1998 "Los cinco sexos". Pp. 79-89 en Transexualismo, transgenerismo y cultura, editado por J. A. Nieto. Madrid: Talasa.

Fraser, N. 1997. Iustitia Interrupta. Reflexiones críticas desde la posición "postsocialista". Santafé de Bogotá: Universidad de los Andes/Siglo del Hombre Editores.

Flaquer, L. 1999. La estrella menguante del padre. Barcelona: Ariel.

Friedan, B. [1963] 1974. La mística de la feminidad. Madrid: Júcar.

Garfinkel, E. 1984. Studies of Ethnomethodology. Cambridge: Polity Press.

Gil Calvo, E. 1997. El nuevo sexo débil. Los dilemas del varón postmoderno. Madrid: Temas de Hoy.

Gilligan, C. 1985. La moral y la teoría. Psicología del desarrollo femenino. México: Fondo de Cultura Económica. 
Goffman, E. 1977. "The arrangement between sexes". Theory and Society 40: 301-331.

Goffman, E. 1979. Gender Advertisments. Nueva York: Harper and Row.

Gregory, A. y J. Windebank. 2000. Women's Work in Britain and France. Practice, Theory and Policy. Londres: Mac Millan.

Guasch, O. 2007. "Los hombres y otras identidades de género". Pp. 323-335 en La Sociología en España, compilado por M. Pérez Yruela. Madrid: Federación Española de Sociología/Centro de Investigaciones Sociológicas.

Hearn, J. 1990. "Child abuse and men's violence". Pp. 63-85 en Taking child abuse seriously: Contemporary issues in child protection theory and practice, edited by The Violence Against Children Study Group. Londres: Unwin Hyman.

Hearn, J. 2002a. "Men, fatherhood and the state: national and transnational perpectives". Pp. 245-272; 293-4 en Making Men into Fathers: Men, Masculinities and the Social Politics of Fatherhood, edited by B. Hobson. Cambridge: Cambridge University Press.

Hearn, J. 2002b. "Nation, state and welfare: the cases of Finland and the UK". Pp. 85-102 en A Man's World: Changing Men's Practices in a Globalized World, edited by R. Pease and K. Pringle. Londres: Zed.

Hochschild, A. y A. Machung. 1989. The Second Shift or the Revolution at Home. Nueva York: Viking.

Ibarra, J. y S. Pinazo. 2008. Mis abuelos me cuidan. Guía para los canguros del siglo XXI. Madrid: ICM.

INE 2003. Encuesta de Empleo del Tiempo. Madrid: Instituto Nacional de Estadística.

INE 2008. Cuenta satélite de los hogares. Madrid: Instituto Nacional de Estadística.

INE 2009. Encuesta de Población Activa, INEbase. Consultado: 3/03, 2009. (www.ine.es).

Irigaray, L. [1974] 1978. Speculum. Espéculo de la otra mujer. Madrid: Saltés.

Irigaray, L. 1981. Le corps-à-corps avec la mère. París: Pleine Lune.

Kane, M. J. 1995. "Resistance/transformation of the oppositional binary: Exposing sport as a continuum" Journal of Sport and Social Issues 19: 191-218.

Kaufman, M. 1998. "The construction of masculinity and the triad of men's violence". Pp. 4-17 en Men's lives, edited by M. S. Kimmel \& M. A. Messner. Boston: Allyn \& Bacon.

Kimmel, M. S., J. Hearn y R. W. Connell. 2005. Handbook on Studies on Men \& Masculinities. Thousand Oaks/Londres/Nueva Delhi: Sage Publications.

Kinsey, A, W. Pomery y C. Martin. [1948] 1998. Sexual Behaviour in the Human Male. Bloomington: Indiana University Press.

Lamarca Lapuente, C. 2004. "La RAE y el monopolio del género ... gramatical", consulta en línea el 7/4/2010: http://www.ucm.es/info/especulo/cajetin/leygener.html. 
Leira, A. 2002. Working Parents and the Welfare State. Cheltenham, Northampton: Edward Elgar.

Lister, R., F. Williams, A. Antonnen, J. Bussemaker, U. Gerhard, J. Heinen, S. Johansson, A. Leira, B. Siim y C. Tobío. 2007. Gendering citizenship in Western Europe. Bristol: The Policy Press.

Maccoby, E. E. 1998. The two sexes: Growing up apart, coming together. Cambridge MA: The Belknap Press of Harvard University Press.

Mead, M. [1935] 2006. Sexo y temperamento en tres sociedades primitivas. Barcelona: Paidós Ibérica.

Mead, M. 1949. Male and female: a Study of the Sexes in a Changing World. Londres: Victor Gollancz.

Messner, M. A. 2005. "Still a Mans's World? Studying Masculinities and Sport". Pp. 313-325 en Handbook on Studies on Men \& Masculinities, edited by M. S. Kimmel J. Hearn y R. W. Connell. Thousand Oaks/Londres/Nueva Delhi: Sage Publications.

Millet, K. [1969] 1995. Política sexual. Madrid: Cátedra.

Nelson, C. 1974. "Public and Private Politics: Women in the Middle East". American Ethnologist 1: 551-563.

Noddings, N. 1995. "Caring". Pp. 7-30 en Justice and Care. Boulder. Colorado: Westview Press.

Osborne, R. y O. Guasch. 2003. Sociología de la Sexualidad. Madrid: Centro de Investigaciones Sociológicas.

Parsons, T. 1978. "La estructura social de la familia". Pp. 31-65 en La familia, edited by E. Fromm et al. Barcelona: Península.

Parsons, T. y R. F. Bales. 1956. Family socialization and interaction process. Nueva York: Routledge.

Pateman, C. 2000. "The Patriarchal Welfare State" Pp. 238-264 en K. Nash (ed) Readings in Contemporary Political Sociology. Oxford: Blackwell.

Pérez Yruela, M. comp. 2007. La Sociología en España. Madrid: Federación Española de Sociología/ Centro de Investigaciones Sociológicas.

Plataforma por Permisos Iguales e Intransferibles de Nacimiento y Adopción (PPIINA), consultado 15/04/2010: (http://www.igualeseintransferibles.org)

Rodríguez Pascual, I. 2008 La infancia en las redes familiares. Sevilla: Instituto de Estadística de Andalucía.

Rosaldo, M. 1979. "Mujer, cultura y sociedad: una visión teórica". Pp. 153-180 en Antropología y Feminismo, editado por O. Harris y K. Young. Barcelona: Anagrama.

Rosaldo, M. 1983. "Moral Analitic Dilemas Posed by the Intersection of Feminism and Social Science". Pp. 76-95 en Social Science as a Moral Inquiry, edited by N. Haah, R. Bellah, P. Rabinow y W. Sullivan. Nueva York: Columbia University Press. 
Rubin, G. 1975. "The Traffic in Women: Notes on the 'Political Economy' of Sex". Pp. 157-210 en Toward an Anthropology of Women, edited by R. Reiter. Nueva York: Monthly Review Press.

Rubin, G. 1984. "Thinking Sex: Notes for a Radical Theory of the Politics of Sexuality". Pp. 267-319 en Pleasure and Danger, edited by C. Vance. Londres: Routledge.

Ruddick, S. 1989. Maternal Thinking. Towards a Politics of Peace. Londres: The Women's Press.

Sacks, K. 1974. "Engels Revisited: women, the organisation of production and private property". Pp. 201-222 en Women, Culture and Society, edited by M. Rosaldo y L. Lamphere. Stanford: Stanford University Press.

Simmel, G. [1921] 1988. Sobre la aventura. Ensayos filosóficos. Barcelona: Península.

Stuart Mill, J. [1869] 2008. La esclavitud femenina. Madrid: Artemisa.

Stuart Mill, J. y H. Taylor. 2001. Ensayos sobre la igualdad sexual. Madrid: Cátedra.

Tobío, C. 2003. "La segregación espacial de género: micro y macroespacios". El valor de la palabra 3:78-103.

Tobío, C. 2005. Madres que trabajan. Madrid: Cátedra.

Tobío, C., M. S. Agulló Tomás, M. V. Gómez y M. T. Martín Palomo. 2010. El cuidado de las personas. Un reto para el siglo XXI. Barcelona: Fundación "la Caixa".

CONSTANZA TOBío es Catedrática de Sociología en la Universidad Carlos III de Madrid. Ha sido profesora visitante en las universidades de Siena, Bath y La Sapienza de Roma, así como en el CNRS de París. Sus áreas principales de investigación son la sociología del género, la relación familia-empleo y las relaciones intergeneracionales. Entre otros libros ha publicado Madres que Trabajan. Dilemas y Estrategias (2005) y El cuidado de las personas. Un reto para el Siglo XXI, así como en las principales revistas de sociología españolas e internacionales.

RECIBIDO: $26 / 08 / 2010$

ACEPTADO: 21/02/2011 